\title{
Efeito da reabilitação vestibular em idosos: quanto ao equilíbrio, qualidade de vida e percepção
}

\author{
Effect of vestibular rehabilitation in elder people: \\ regarding balance, quality of life and perception
}

M agali Peres ${ }^{1}$

Elaine da Silveira ${ }^{2}$
${ }^{1}$ Universidade Luterana do Brasil. Av. Farroupilha 8.001, Bairro São José. 92425-900 Canoas RS. magali.peres@hotmail.com

${ }^{2}$ Programa de Saúde

Coletiva, Universidade Luterana do Brasil.
Abstract The current concerns with quality of life, population aging, limitationsimposed by balance disturbance in the elder population and the alternative treatment through the vestibular rehabilitation had justified this study. The objective was to analyze in perspective the application of the Cawtome \& Cooksey protocol for vestibular rehabilitation results in aged institutionalized people with complains of balance alterations and probability of fall, aiming the improvement of quality of life and that elders could perceive the limitations imposed by the balance disturbance. A hybrid methodology was used, searching an integration of quantitative and qualitative data associating the experimental to the phenomenological in a population of 30 elders with complains of balance alterations living in an asylum. The evaluation instruments had been the Berg Scale, Dizziness $\mathrm{H}$ andicap I nventory (DHI) and speech analysis. As conclusion, it was evidenced that, within the population studied, the vestibular rehabilitation was effective regarding the improvement of symptoms of balancealteration in relation to quality of life, probability of fall as well as the security and independence feelings of the elders.

Key words Dizziness H andicap Inventory (DHI), Balance, Berg Scale, Elder people, Quality of life, Vestibular rehabilitation
Resumo As atuais preocupações com qualidade devida (QV), envelhecimento da população, limitações impostas pelos distúrbios do equilíbrio na população idosa ea alternativa de tratamento por meio da reabilitação vestibular (RV) justificaram este estudo. Nele, objetivou-se analisar, perspectivamente, os resultados da aplicação do protocolo de Cawtome \& Cooksey para RV, em idosos institucionalizados, com queixas de alterações de equilíbrio e probabilidade de queda $(P Q)$, visando à melhoria da QV e à percepção desses idosos sobre as limitações impostas pelos distúrbios do equilíbrio. U tilizou-se uma metodologia híbrida, buscando a integração de dados quantitativos e qualitativos, associando-seo estudo experimental ao fenomenológico, em uma população detrinta idosos asilados com queixas de alterações do equilíbrio. Os instrumentos de avaliação foram a Escala de Berg, Dizziness $\mathrm{H}$ andicap Inventory (DHI) eanálise de conteúdo. Como conclusão do estudo, constatou-se que, na população estudada, a RV foi efe tiva quanto à melhora dos sintomas de alteração do equilíbrio em relação à QV, à $P Q$ e aos sentimentos de segurança e independência dos idosos. Palavras-chave Dizziness H andicap Inventory (DHI), Equilíbrio, Escala de Berg, I dosos, Qualidade de vida, Reabilitação vestibular 
Introdução

A sociedade atual é paradoxalmentevoltada para o jovem, cuja juventude é admirada e sinônimo de valor, mérito e promessa. Por isso, a idade de muitos é escondida eo rejuvenescimento éo sonho de todos. N essa sociedade altamente competitiva, os idosos têm de lutar para sobreviver, apesar de seus declínios físicos e mentais, resultantes do envel hecimento ${ }^{1}$.

0 prolongamento da expectativa de vida leva a uma mudança nos padrões epidemiológicos da população, marcada pela diminuiçã̃o da mortalidade e das taxas de natalidade, determinando novas necessidades de atenção à saúde. D evido às alterações provocadas pelo envelhecimento, estão cada vez mais presentes, na população, os agravos relacionados aos distúrbios do equilíbrio².

Sabe-sequeo envelhecimento comprometea agilidade, o sistema nervoso central, por causa da degeneração e perda progressiva de células nervosas no sistema vestibular periférico e central, processo responsável pela ocorrência devertigem (sensação de rotação corporal ou do ambiente), tontura (desequilíbrio sem sensação rotatória) e desequilíbrio na população geriátrica². As quedas dos idosos têm relação causal com $12 \%$ de todos os óbitos, sendo responsáveis por $70 \%$ das mortes acidentais em pessoas acima de 75 anos. Para aquel es que necessitam de hospitalização, o risco de morte varia entre $15 \%$ e 50\%. Os fatores de risco que predispõem os idosos a esse agravo estão relacionados aos efeitos cumulativos das alterações relacionadas a idade, doenças e ambiente inadequado. Tais fatores englobam as alterações fisiológicas do processo de envelhecimento como diminuição da visão; diminuição da audição; distúrbios vestibulares; distúrbios proprioceptivos ${ }^{3}$. D esse modo, as atuais preocupações com qualidade de vida, enveIhecimento da população e as limitações impostas pelos distúrbios do equilíbrio, especialmente na população idosa, justificam esta pesquisa.

Como alternativa de tratamento desses agravos, há medicação e exercícios específicos para a reabilitação vestibular (RV), tratamento terapêutico que tem por objetivo a promoção da melhora do equilíbrio global e a restauração da orientação espacial para o mais próximo do fisiológico. A RV busca, por meio de exercícios, estimular os fenômenos de adaptação, envolvendo a habituação e a compensação ${ }^{2}$. Também visa estimular a estabilização visual (nitidez do campo visual), aprimorar a interação visuovestibular, promover orientação espacial adequada e reduzir desconforto durante a movimentação da cabeça, bem como propiciar maior estabilidade da postura corporal, no repouso e na movimentação, principalmente nas situações que podem desencadear maior conflito sensorial ${ }^{4}$.

Os resultados obtidos e publicados pela literatura mundial, conforme relatam Bittar e Pedalini ${ }^{5}$, vêm incentivando os pesquisadores a buscarem a utilização do método de RV, considerado uma excel ente opção terapêutica para os pacientes portadores de vestibulopatias porque, além de melhorar sobremaneira o equilíbrio do doente, tem função profilática, ajudando- 0 a restabelecer a confiança em si mesmo, reduzindo a ansi edade e melhorando o convívio social ${ }^{5}$.

Infelizmente, apesar da existência dos protocolos de RV, as atuais instituições e os programas de saúde voltados para o idoso, comumente, não apresentam a RV como uma opção de tratamento acessível eadequadamenteacompanhada. Exatamente por isso, este estudo propõe, por meio de uma pesquisa híbrida, associando um estudo experimental a um fenomenológico, analisar, prospectivamente, os resultados da aplicação do protocolo de Cawtome \& Cooksey para a RV, em idosos institucionalizados, avaliados quanto às alterações de equilíbrio, probabilidade de queda $(P Q)$, melhoria da qualidade de vida (QV) e percepção deles sobre as limitações impostas pelos distúrbios do equilíbrio. Assim, pretende-se destacar a importância desse recurso para a reabilitação dessas pessoas que, em razão de problemas característicos da idade, tornam-se marginalizadas por uma sociedade que vê a juventude como um estágio de vida ideal e que deve ser mantido.

\section{M ateriais e métodos}

Buscou-se uma integração de dados quantitativos e qualitativos, dentro de um mesmo estudo, por meio da pesquisa intervencionista (experimental) e qualitativa (fenomenológica). A dicotomia entre dados representa a principal distinção epistemológica emetodológica entreas ciências sociais e comportamentais. Essa mescla criteriosa enriquece a pesquisa.

Vale acrescentar que o delineamento integrado traz vantagens como a complementação, evitando, possivelmente, as limitações da abordagem única; o incremento, visto que forma elos entre duas direções do conhecimento; a maior validade favorecida, já que possui dados múltiplos; a criação de novas fronteiras, expandindo a linha de investigação ${ }^{6}$. 
0 presente estudo foi desenvolvido em uma instituição de idosos da região metropolitana de Porto Alegre(RS), que recebe idosos sem dependência para as atividades de vida diária. Essa instituição comporta uma população de 60 pessoas, sendo 43 mulheres e 17 homens, com idades entre 60 e 94 anos. A partir de uma entrevista censitária, foram arrolados idosos que referiram queixas de alterações do equilíbrio, população que totalizou trinta indivíduos.

0 grupo em estudo foi selecionado a partir de uma entrevista iniciada com uma descrição sobre as características e os sintomas relativos aos distúrbios de equilíbrio. A pós o esclarecimento, os idosos foram questionados sobre a presença desses sintomas. Os pacientes com queixas de alterações do equilíbrio foram alocados para uma intervenção com aplicação de um programa de RV. Em seguida, utilizou-se uma pesquisa com abordagem fenomenológica, por meio da técnica Grupo Focal, com a intenção de observar a percepção dos pacientes quanto às limitações impostas pelos distúrbios do equilíbrio.

Esses idosos, após o esclarecimento sobre 0 estudo, aceitaram participar do experimento, pois não realizavam, atéo momento, nenhum tipo de tratamento para o problema em questão. D o total de trinta idosos, apenas 21 concordaram em realizar o programa de exercícios baseado no protocolo de Cawtome \& Cooksey, o qual consiste em movimentos rotatórios, desvios laterais, desvios cefalocaudais, de marcha, realizados em ortostase e na posição sentada, com e sem auxílio visual, compondo o Grupo Experimental (GE). Os outros nove indivíduos que não aceitaram efetuar os exercícios formaram o Grupo Controle (GC). 0 tempo das sessões de reabilitação vestibular foi de sessenta minutos, sendo realizadas duas vezes por semana durantenoventa dias ${ }^{4}$.

Foram aplicados aos participantes dois instrumentos de avaliação quantitativa: a Escala de Equilíbrio deBerg (EEB) e o Dizziness H andicap Inventory (DHI) brasileiro, antes e após a intervenção. Os grupos focais foram realizados apenas com o grupo submetido às sessões de RV.

A Escala de Equilíbrio de Berg (EEB) é um instrumento que mensura as habilidade de equilíbrio do indivíduo ao realizar determinados testes. Os escores absolutos são obtidos por meio de 14 testes avaliando a habilidade do indivíduo de se sentar, ficar de pé, alcançar, girar em volta de si mesmo, olhar por cima de seus ombros, ficar sobre apoio unipodal e transpor degraus. A pontuação total é de 56, eíndiceigual ou menor a 36 está associado a 100\% de risco de quedas.
Cada testetem cinco opções de classificação, sendo que zero significa a inabilidade de desenvolver a ação solicitada. As opções aumentam até 4, conforme aumenta a habilidade total de exercer a função. Dessa forma, quanto maior o escore, melhor a condição de equilíbrio do idoso e menor o seu risco de queda?

Os escores obtidos na EEB são transformados em percentual de PQ . A pontuação total éde 56 , e índice igual ou menor a 36 está associado a 100\% de risco de queda. Esses escores foram aplicados para se obter o índice de Possibilidade de Queda (PQ) pela seguinte equação: 100\% x exp (10,46$0,25 \times$ BBS escore $+2,32 \times$ história deinstabilidade) / [ $1+\exp (10,46-0,25 \times$ BBS escore $+2,32 \times$ história de instabilidade)], sendo que BBS escore é 0 escore obtido pelo indivíduo na EEB. Quanto à história de instabilidade, é atribuído o valor zero quando não há relato de instabilidade, e o valor 1 quando há essa ocorrência. N este estudo, todos os indivíduos relataram instabilidade, sendo este um critério para seleção da população da pesquisa7.

Esse instrumento mostra excelente confiabilidade $(0,96)$ e de moderada para boa correlação com outros instrumentos de avaliação funcional do equilíbrio, como: Escala de Mobilidade de Barthel, 0,67; Teste do "Up and Go", 0,76; Escala do Equilíbrio de Tinetti, 0,91. Esta escala tem excelenteobjetividade deteste reteste $(\text { ICC }=0,98)^{7,8}$.

$0 \mathrm{DHI}$ brasileiro avalia o prejuízo quanto à $Q V$, devido às alterações do equilíbrio, verificando a interferência de tontura ou vertigem nos aspectos físicos, funcionais e emocionais do paciente, na realização das atividades de vida diária. Por meio de 25 questões, transforma os comprometimentos de QV em um escore para cada aspecto e um escore total ${ }^{8}$.

Para que fique claro o significado dos resultados, éimportante saber que o DHI écomposto deperguntas com três opções derespostas ( "sim", "não" e "às vezes"), sendo que a primeira opção representa um escore de 4 e, sequencialmente, zero e2. Dessa forma, quando determinada atividade pode trazer 0 aumento do problema, se positivo, representa o escore 4; se negativo, zero; se eventualmente, 2. Assim, quanto menor o escore individual ou total, menores são as limitações e melhor a QV dos indivíduos.

Os dados iniciais e finais foram analisados e comparados pelo teste T-Student porque as variáveis analisadas passaram no teste de aderência de Kolmogorov-Smirnov. 0 teste T-Student exigequeas variáveistenham distribuição deprobabilidade normal. 0 nível de significância determinado foi de $5 \%$, ou seja, $p<0,05$. 
A investigação fenomenológica trouxe a possibilidade de se refletir sobre as experiências da vida das pessoas, pois descreve os sentimentos que elas experimentam em relação a um fenômeno. N esta pesquisa, os idosos vivenciam as limitações em relação aos distúrbios do equilíbrio antes e após a intervenção.

O pesquisador fenomenológico pergunta: "Qual a essência desse fenômeno experimentado por essas pessoas e o que ele significa?" N este estudo, perguntou-se: "Qual a essência dos distúrbios do equilíbrio experimentados por essa população idosa e que significados eles têm nas suas vidas?"

A principal fonte de dados neste estudo foi a conversação em profundidade entre o pesquisador e o informante como coparticipantes totais, efetuada por meio da técnica de GF (Grupo Focal). A técnica em questão valoriza a comunicação entre os participantes da pesquisa, utilizando a interação do grupo para fornecer tipos distintos de dados. Isso significa que, em vez de o pesquisador fazer perguntas a cada pessoa, os indivíduos foram estimulados a interagir, a perguntar, a trocar histórias e a comentar as experiências e os pontos de vista uns dos outros ${ }^{9}$.

0 método utilizado para análise qualitativa de conteúdo foi o de Bardin ${ }^{10}$, que, mediante a análise da comunicação verbal, visa sistematizar e objetivar o conteúdo das mensagens, utilizando ou não indicadores quantitativos. N esta pesquisa, foram utilizados os relatos sistematizados, segundo a frequência das falas, em categorias relativas à independência e segurança.

Esses grupos focais foram realizados em três momentos diferentes do desenvolvimento da pesquisa, ou seja, no início, no meio e após finalizar as sessões de RV. Assim, foram denominados GF1, GF2 eGF3, respectivamente, com os 21 indivíduos que se propuseram a participar da intervenção.

As reuniões foram realizadas com registro audiovisual. A partir daí, foram transcritas as falas e classificadas, segundo análise de conteúdo, em categorias referentes à segurança e independência dos idosos.

\section{Consideraçõeséticas}

Foram observados todos os requisitos para atender aos critérios éticos. 0 projeto foi aprovado pelo ComitêdeÉtica em Pesquisa da Universidade Luterana do Brasil (Ulbra).

\section{Resultados}

Os resultados são apresentados, primeiramente, quanto ao estudo experimental, no qual consta 0 escore obtido pela Escala de Berg, a PQ e o DHI brasileiro. No segundo momento, são descritos os resultados do estudo fenomenológico por meio da análise do discurso dos idosos entrevistados.

\section{Resultados do estudo experimental}

Os resultados da Escala de Equilíbrio de Berg (EEB), avaliada nos GE e GC no início e no final dos procedimentos propostos, foram obtidos em um período de noventa dias, conforme os resultados indicados no Gráfico 1.

Observando-se o Gráfico 1, verificam-se diferenças nos escores médios antes e após a intervenção, referente a cada um dos testes.

No Grupo Controle (GC), comparando-se os escores médios antes eapós a intervenção, não foram obtidas diferenças significativas, ou seja, o grupo que não participou dos exercícios de RV não teve alterações nos testes de equilíbrio propostos pela EEB, conforme o Gráfico 1 .

Os resultados obtidos em média no GE e no GC estão ilustrados no Gráfico 2:

O Grupo Experimental obteve um escoreinicial de $84 \%$ de $P Q$, considerando a média dos indivíduos participantes do grupo. A pós a intervenção, houve um decréscimo no valor de $P Q$, com uma diferença de $22,99 \%$, ou seja, passou para $61,58 \%$ ao final da intervenção. H ouve, então, uma diferença estatisticamentesignificativa.

No Grupo Controle (GC), os valores relativos aos escores médios de $\mathrm{PQ}$ foram inicialmente $91,77 \%$ e, ao final, $91,69 \%$, ou seja, não sofreram alterações. Assim, esses idosos estão sujeitos aos mesmos riscos de queda do início do estudo.

$\mathrm{O}$ DHI éum instrumento que avalia a interferência da tontura na qualidade de vida dosidosos quanto aos aspectos físicos, emocionais e funcionais. N esteestudo, obtiveram-seos seguintes resultados, conforme o Gráfico 3.

Quanto ao grupo submetido à intervenção, conforme o Gráfico 3, observam-se os escores médios obtidos antes e após a intervenção, referentes ao DHI. Nos itens do aspecto físico, o escore em média, no início da pesquisa, era de 15,62; após a intervenção, houveum decréscimo para 6,86.

No escore emocional médio, a mudança decrescente foi de 17,61 para 7,52; assim como a média do aspecto funcional, teve uma redução de 22,77 para 9,45 após a intervenção. 


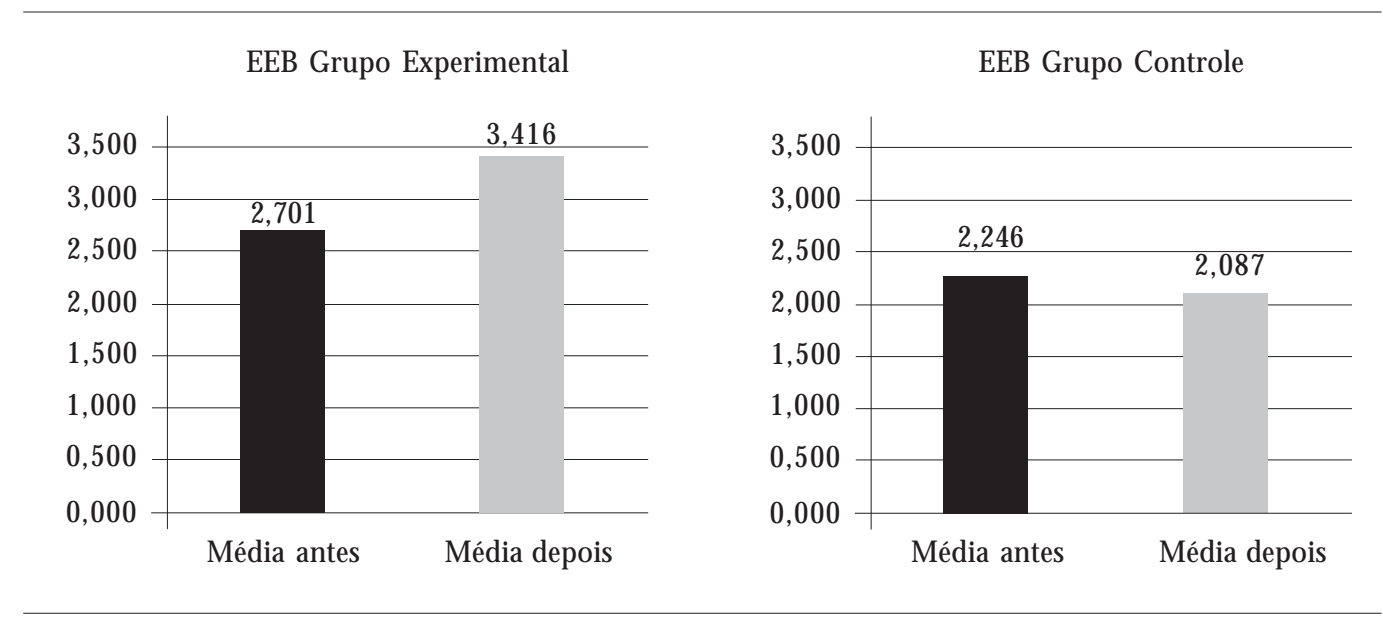

Gráfico 1. Escores médios da EEB no Grupo Controle e no Grupo Experimental em idosos, 2005.

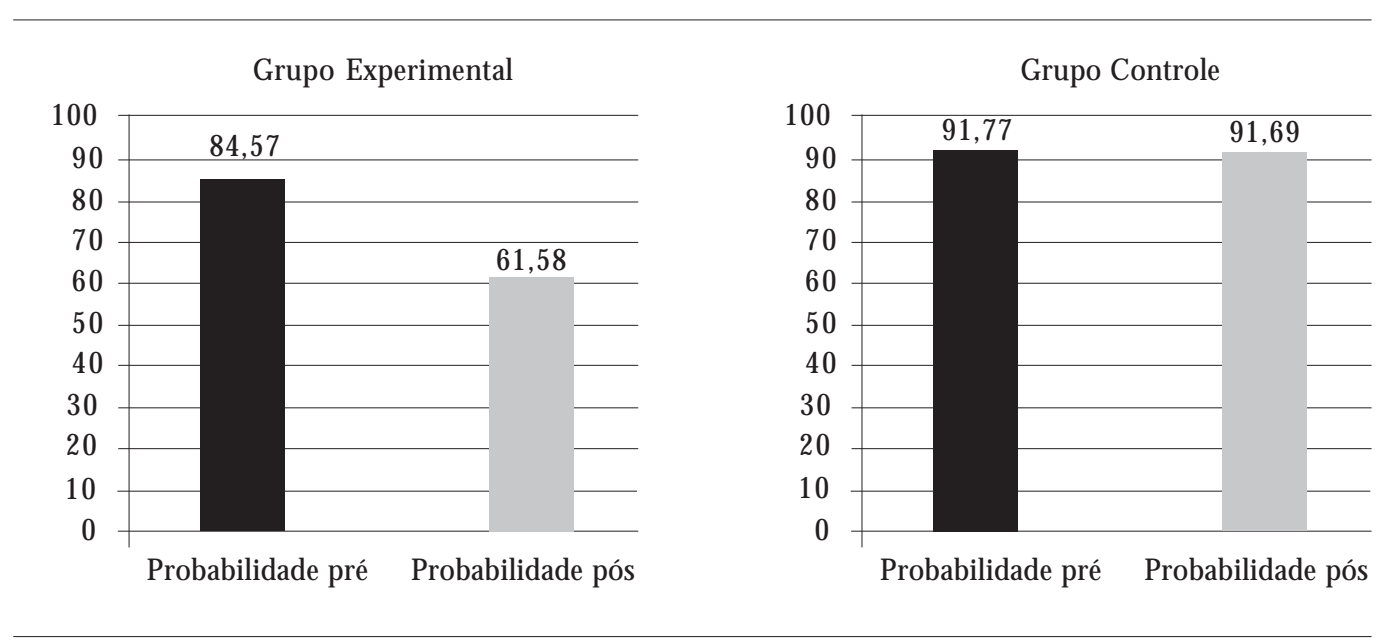

Gráfico 2. Percentuais relativos à probabilidade de queda (PQ) no Grupo Experimental e no Grupo Controle, 2005.

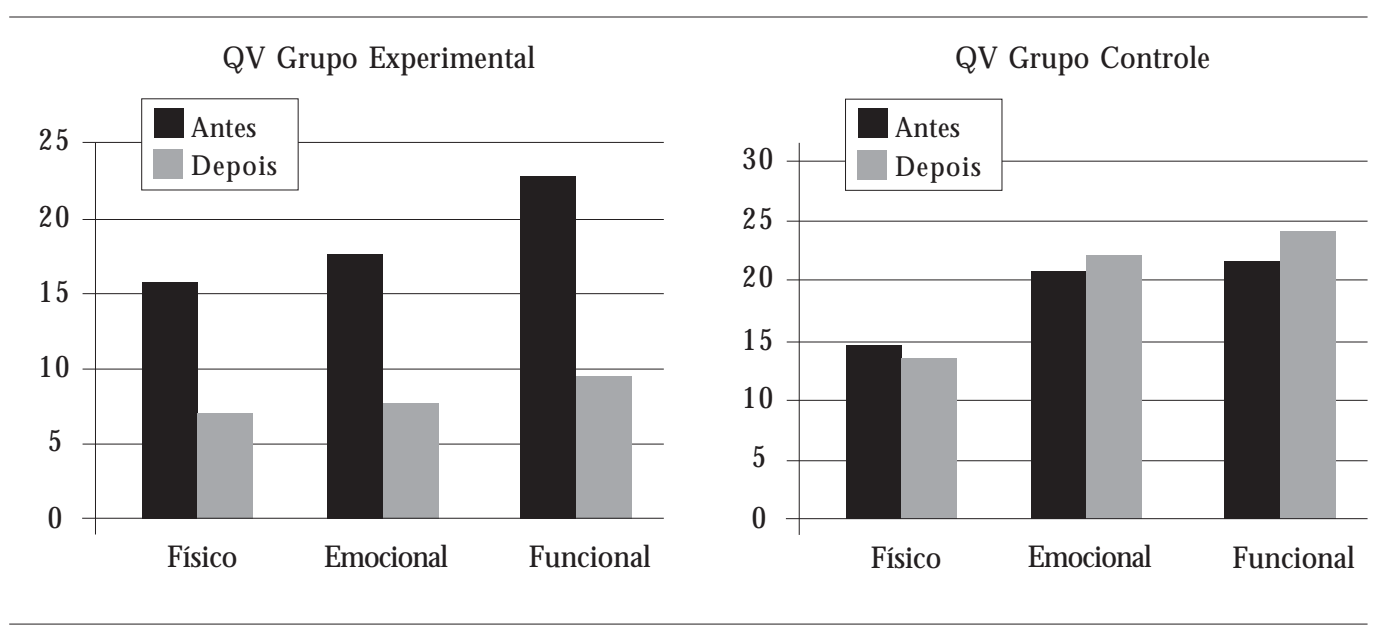

Gráfico 3. Escore referente ao DHI brasileiro antes e após a intervenção no Grupo Experimental e no Grupo Controle nos idosos, 2005. 
A soma dos três aspectos, tanto antes como após a aplicação do protocolo de RV, foi um escore total, obtendo-se, em média, uma diferença de 32 pontos, ou seja, de 56 para 23,86 pontos. Assim, constatou-se o declive nos três itens, um decréscimo dos escores, representando a evolução quanto à qualidade de vida estatisticamente significativa nos idosos.

Verificou-se no GC que os escores obtidos no início da pesquisa quanto ao aspecto físico, em média, foram 14,67 pontos e, após, 13,55 pontos, o que resultou em escore não significativo estatisticamente, apesar de este, em média, ter diminuído. No aspecto emocional, as médias foram de 20,67 pontos e, após, 22 pontos, ou seja, o escore aumentou, o que representa menor QV dosindivíduos nesse aspecto. Quanto ao funcional, também houve um aumento das limitações e redução da QV, obtendo-se, em média, 21,56 e, após, 24,23 pontos, representando uma mudança para pior, estatisticamente comprovada.

\section{Resultados do estudo fenomenológico}

No eixo fenomenológico deste estudo, por meio da análise de conteúdo das falas dos três grupos focais, realizados no início, no meio eno final da intervenção, obtiveram-se informações referentes à questão temática: qual a essência dos distúrbios do equilíbrio experimentados por essa população idosa e que significados eles têm nas suas vidas?

A metodologia qualitativa foi utilizada para buscar a compreensão das limitações causadas pelos distúrbios do equilíbrio em idosos, tendo como premissa a difícil mensuração da tonturae das demais limitações impostas pelos distúrbios do equilíbrio. Porém, eles podem causar limitações importantes eatélesões irreversíveis aos seus portadores.

As falas de cada grupo foram agrupadas se gundo o número de vezes em que apareciam relatos de vivências ou sentimentos relativos a essas categorias, obtendo-se, assim, os resultados apresentados na Tabela 1.

O bservou-se que as expressões utilizadas em cada grupo, referentes a questões de segurança e independência, distribuíram-se, quanto à frequência, em diferentes formas. No Grupo Focal I, sobressaíram os sentimentos relacionados à insegurança eà dependênciafísica, principalmente por meio da repetição de palavras como "medo" e "insegurança" eda frequência com que são relatados episódios pelos idosos, como: A gente não tem equilíbrio, então a gente nunca sabe pra que
Tabela 1. Frequência de relatos de vivências e sentimentos dos três GF, de acordo com as categorias de segurança e independência em idosos, 2005.

\begin{tabular}{lccc}
\hline \multicolumn{1}{c}{ Grupos } & $\begin{array}{c}\text { Grupo } \\
\text { Focal I }\end{array}$ & $\begin{array}{c}\text { Grupo } \\
\text { Focal II }\end{array}$ & $\begin{array}{c}\text { Grupo } \\
\text { Focal III }\end{array}$ \\
\hline Insegurança & 64 & 9 & 2 \\
Dependência & 66 & 7 & 7 \\
Segurança & 0 & 23 & 26 \\
Independência & 0 & 21 & 19 \\
\hline
\end{tabular}

lado vai, então dá medo, mete muito medo na pessoa; tontura limita em quase tudo; a gente se sente como uma pessoa perdida.

No Grupo Focal II, observaram-se como característica predominante as falas que expressaram sentimentos de segurança e independência, relacionadas à atividade física, ao lazer e à vida social, como: quando eu danço, quedas, às vezes elas ameaçam, mas eu consigo ligeiro me livrar delas; uns tempos atrás eu tive muito desequilíbrio, se eu começasse a cair, por qualquer coisa eu ia embora, não tenho mais essas quedas. As palavras medo einsegurança estiveram presentes, mas em menor escala.

Já o Grupo Focal III foi marcado pelo aparecimento de expressões de satisfação e evolução, demonstrando que houve sentimento de evolução dos sintomas inicialmente relatados. Registraram-se os depoimentos: 0 exercício é bom, é ótimo; meu equilíbrio, tudo bom; mel horei; tô mais alegre; senti mais firmeza pra caminhar; Consegui mais coragem pra caminhar sozinha.

\section{Discussão}

Este estudo não se limita a confirmar que a RV é um procedimento efetivo para os distúrbios do equilíbrio também na população idosa. Pretende acrescentar o enfoque da QV em idosos no âmbito da Saúde Coletiva.

Conforme foi descrito por Lobos ${ }^{11}$, em um estudo sobre a RV em grupo de idosas com idade igual ou superior a 60 anos, a RV éde baixo custo, mas foi efetiva em produzir modificações no equilíbrio e na habilidade funcional das idosas, podendo ser aplicada na promoção da saúde e na melhora da qualidade de vida dos idosos.

Segundo a escala EEB, aplicada no GE do estudo, houve uma evolução nas habilidades para execução dos testes relativos ao equilíbrio estáti- 
Co e dinâmico. Como na EEB quanto maior 0 escore melhor a condição de equilíbrio e menor probabilidade de queda (PQ), verificou-se que os idosos desse grupo tiveram diminuição de $84,6 \%$ para $61,6 \%$, com uma diferença de $23 \%$. Esses dados corroboram os achados de Ribeiro e Pereira ${ }^{7}$, que, por meio deum experimento utilizando o mesmo protocolo, também obtiveram diminuição da PQ com uma diferença de $30,4 \%$, considerando antes e após a interven ção no GE. A diferença encontrada entre os escores dos dois estudos provavelmente está relacionada às características da população submetida à pesquisa em questão, composta por idosos com queixa de distúrbios do equilíbrio - Ribeiro e Pereira ${ }^{7}$ partiram de uma população de idosos saudáveis.

Para as habilidades que não demonstraram evolução significativa no GE, como "apanhar objeto no chão", por exemplo, acredita-se que sedeva à complexidade do movimento que envolve um conjunto de exigências visuais, proceptivas esensoriais integradas. Acredita-se que, para a obtenção da evolução dos sintomas mais resistentes, seria necessário maior número de sessões, ou até associação de outras técnicas de tratamento.

0 aumento no escore do EEB e a redução da probabilidade de queda interfere diretamentena prevenção da morbimortalidade por quedas, que representa $12 \%$ de todos os óbitos na população geriátrica do Brasil ${ }^{3}$.

Segundo Pereira et al. ${ }^{3}$, a ocorrência de queda anual por faixa etária no Brasil é de $67 \%$ entre indivíduos de 65 a 84 anose $51 \%$ acima de 85 anos. A população brasileira envelhece a passos largos, e a participação das quedas na mortalidade proporcional por causas externas cresceu de 3\% para $4,5 \%$, nos últimos anos, nessa faixa etária.

Quanto aos escores de EEB e PQ , o GC não apresentou diferenças significativas, o que demonstra que os resultados obtidos neste estudo, no GE, estão associados à interven ção proposta, já que os dois grupos foram formados por indivíduos com características semelhantes, moradores de uma mesma instituição, mesma faixa etária ecom rotinas semelhantes. Esses achadostambém foram comparados ao estudo de Ribeiro e Pereira ${ }^{7}$, pois seus resultados mostraram que 0 Grupo Controle não apresentou diferenças significativas entre as médias naEEB, na primeira ena segunda avaliações, nem entre as médias da PQ .

Comparando-se os escores de EEB e PD do GE e do GC, antes e depois da intervenção, verificou-se que o GE e o GC, quando tiveram os seus escores de EEB ePQ comparados, não apresentaram diferenças significativas antes; porém, essas diferenças foram estatisticamente comprovadas após o experimento.

0 instrumento utilizado neste estudo para avaliar a QV com alteração de equilíbrio, o DHI, mostrou resultados no GE que demonstraram evolução significativa em todos os aspectos, considerando a avaliação antes e pós-intervenção.

$\mathrm{N}$ o aspecto físico, a diferença entreos escores foi de 8,76 pontos. No emocional, foi de 10,09 pontos e, no funcional, de 13,32 pontos. Assim houve, de modo geral, diferenças importantes, em especial quanto ao aspecto funcional, que avalia atividades como lazer, trabal hos e atividades de vida diária (AVDs), as quais requerem capacidade de autonomia do idoso no contexto social. Esse aspecto é muito importante para a qualidade de vida dele, visto queinterfere na dependência do indivíduo no contexto social, conforme relatam N egri et al. ${ }^{12} \mathrm{em}$ seu estudo sobre a previsibilidade de agravos na população idosa, associando o risco de agravos e fragilidade do idoso à dependência funcional.

Muitos estudos utilizaram para avaliação os resultados da RV eo de QV DHI, como éo caso dos autores indicados a seguir. Resende et al. ${ }^{2}$ constataram que o grupo querealizou a RV apresentou melhora significativa na qualidadedevida, o que não ocorreu no GC. Ganança et al. ${ }^{13} \mathrm{ePe}$ dalini eBittar ${ }^{14}$ ressaltaram quea reabilitação vestibular é o melhor tratamento terapêutico para pacientes acometidos de disfunção vestibular e pode ser usada com ou sem auxílio de medicamentos.

Silveira et al. ${ }^{15}$, em experimento com três grupos durante dez semanas consecutivas de tratamento, submeteram um deles à RV por meio do protocolo Cawthorne \& Cooksey. Os resultados mostraram uma redução estatisticamente significativa do handicap, provocada pelas alterações vestibulares, tanto na pontuação total como nas variáveis física, funcional e emocional. Também os estudos de Cowand et al. ${ }^{16}$ sobre a eficácia da RV, por meio do DHI, obtiveram diferenças significativas nos escores antes e após a intervenção, nos três aspectos.

M ruzek et al. ${ }^{17}$, por meio de uma randomização, constataram que um programa de RV trouxe muitos benefícios aos pacientes, podendo ser associado a reforço social. Krebs et al. ${ }^{18}$, em estudo com uma população com idade média de 64 anos, observaram que o GE, após oito semanas deRV, apresentou evolução significativa, enquanto a diferença do GC foi de $1 \%$ no escore de QV.

Topuz et al. ${ }^{19}$, em estudo sobre a RV em disfunção vestibular crônica, obtiveram um decrés- 
cimo no escore nas duas primeiras semanas de tratamento. Corna et al. ${ }^{20}$, em estudo comparando o protocolo de exercícios de Cawthorne\& Cooksey com tratamento medicamentoso, concluíram que este promoveu incremento das alterações de equilíbrio e incremento da QV.

Badke et al. ${ }^{21}$ constataram, por meio do DHI, uma evolução das alterações vestibulares em pacientes submetidos à RV. Albera et al. ${ }^{22}$, em estudo randomizado, obtiveram maior escore no tratamento por meio da RV do que no medicamentoso. Ganança et al. ${ }^{8}$ afirmaram que estudos mostraram a importância de se avaliarem os prejuízos da QV em pacientes vertiginosos com o intuito de quantificar os efeitos impostos pela vertigem nas funções de vida diária, além de auxiliar na escolha do tratamento e na sua avaliação.

Esses estudos demonstraram a efetividadedos programas de RV quanto à melhoria da $\mathrm{QV}$ em idosos, tornando-a uma importante alternativa não só terapêutica como também de promoção de saúde para os indivíduos que sofram desse agravo. Por meio da realização dos exercícios de $\mathrm{RV}$ periodicamenteem grupo, obtém-setambém uma melhora na relação social, aumentando a autoestima, fazendo-os perceber que os distúrbios de equilíbrio são comuns no idoso e que podem deixar de ser incapacitantes.

Conforme relatado por Lobos ${ }^{11}$, a RV supervisionada traz confiança, segurança e motivação, assim como a interação entre pacienteeterapeuta influencia no resultado do tratamento. Por esse motivo, échegado o momento de ir além da descrição, basicamente quantitativa, das mudanças relativas à intervenção em uma população, obtendo assim um melhor entendimento dessa problemática. Para tanto, é necessária uma análise das experiências e dos sentimentos dos idosos envolvidos.

O GF1, em relação à categoria de insegurança, apresenta 64 afirmações de vivências e sentimentos negativos, ou seja, expressão de insegurança, com 66 falas relativas à dependência e nenhum relato vinculado a sentimentos de segurança.

No segundo grupo, realizado no 120 encontro no processo de aplicação do protocolo, ainda prevaleciam noverelatos desentimentos evivências de insegurança e sete de dependência, verificando-se assim uma evolução, pois foram identificados 23 relatos de segurança e 21 de independência.

No último GF, houve um marcante predomínio de falas interpretando sentimentos de segurança eindependência, totalizando 45 afirmações, enquanto persistiam apenas nove relatos de experiências negativas em relação a essas categorias.

$V$ isto que os sentimentos de segurança e independência são essenciais para a QV na população em estudo, acredita-se que o incremento dos programas de RV em caráter preventivo ou curativo pode colaborar para atingir a meta de QV na terceira idade.

Conforme relatado por Paiva et al. ${ }^{23} \mathrm{em}$ um artigo referente à associação da tontura a sintomas psicológicos, concomitantes à queixa devertigem vêm a angústia, a ansiedade, o medo, a depressão e os distúrbios de memória. Dentre estes, a angústia foi o sintoma psicológico de maior prevalência.

\section{Conclusão}

Com base no estudo apresentado, verifica-seque a gravidade potencial dos distúrbios do equilíbrio nos idosos confere à prevenção um lugar privilegiado, porque uma queda pode ser considerada um evento sentinela na vida de uma pessoa idosa, um marcador potencial do início de um importante declínio funcional ou um sintoma de uma patologia nova, em razão da perda de capacidades do corpo.

Quanto aos instrumentos utilizados, a EEB foi importante para que as pesquisadoras pudessem distinguir os efeitos quanto às alterações de equilíbrio eà probabilidade de queda. $0 \mathrm{DHI}$, por sua vez, permitiu distinguir os efeitos da RV na QV, tornando-se possível diferenciar os efeitos do GE e do GC. Portanto, neste estudo, constatou-se uma efetiva melhora dos sintomas de alteração do equilíbrio quanto à QV, probabilidade de queda e sentimentos de segurança e independência dos idosos da população em estudo após a RV.

O setor responsável pela saúde deve estar ciente de que o desafio maior no século XXI, no Brasil, será cuidar de uma população de mais de 32 milhões de idosos, fazendo frente a uma crescente demanda por procedimentos diagnósticos eterapêuticos das doenças crônicas, além deuma demanda ainda maior por serviços de reabilitação física e mental. Será preciso estabelecer indicadores de saúde capazes de identificar idosos de al to risco de perda funcional eorientar açõesconcentradas de promoção de saúde e manutenção da capacidade física. 


\section{Colaboradores}

M Peres foi responsável pela concepção, pesquisa e redação final do artigo; E Silveira foi a orientadora da pesquisa e também responsável pela elaboração do artigo.

\section{Referências}

1. Fleck M, Louzada $M$, Xavier $M$, Chachamovich $E$, Vieira G, Santos L et al. Aplicação da versão em português do instrumento abreviado de avaliação da qualidade de vida WHOQOL-bref. Rev Saude Publica 2000; 34(2):178-183.

2. Resende CR, Taguchi, CK, Almeida JG, Almeida J, Fujita R. Reabilitação vestibular em pacientes idosos portadores de vertigem posicional paroxística benigna. Rev Bras Otorrinolaringol 2003; 69(4):535-540.

3. Pereira SEM, Buksman S, Perracini MPL, Barreto $K M L$, Leite VMM. Projeto Diretrizes. Associação M édica Brasileira e Conselho Federal de M edicina. Sociedade Brasileira de Geriatria e Gerontologia 2001; São Paulo. [acessado 2008 jun]. Disponível em: http:/ /www. projetodiretrizes.org.br/projeto_diretrizes/ 082.pdf

4. Ramos S, Ramos R, Caovilla H. Reabilitação vestibular. In: Campos C, Oliva H. Tratado de Otorrinolaringologia. São Paulo: Roca; 2002.

5. Bittar R, Pedalini M. Por que a reabilitação vestibular falha? Archives of Otorhinolaryngology 2000; 4(1):118-122.

6. Polit DF, Beck CT, Hungler BP. Fundamentos de pesquisa em enfermagem. $5^{a}$ ed. Porto Alegre: Artmed; 2004.

7. Ribeiro ASB, Pereira JS. M elhora do equilíbrio e redução da possibilidade de queda em idosas após os exercícios de Cawthorne e Cooksey. Rev Bras Otorrinolaringol 2005; 12(2):113-122.

8. Ganança FF, Castro AS, Branco FC, Natour J. Interferência da tontura na qualidade de vida de pacientes com síndrome vestibular periférica. Rev Bras Otorrinolaringol 2004; 70:17-26.

9. Kitzinger J. Grupos focais com usuários e profissionais da atenção básica. In: Pope C, M ays N. Pesquisa qualitativa na atenção à saúde. $2^{\mathrm{a}}$ ed. Porto Alegre: Artmed; 2005.

10. Bardin L. Análise de conteúdo. Lisboa: Edições 70; 1979.

11. Lobos M B. U ma proposta de reabilitação vestibular em grupo para idosas institucionalizadas [dissertação]. Santa M aria (RS): Universidade Federal de Santa M aria; 2002.

12. Negri LSA, Ruy GF, Collodetti JB, Pinto LF, Soranz DR. Aplicação de um instrumento para detecção precoce e previsibilidade de agravos na população idosa. Cien Saude Colet 2004; 9(4):33-46.

13. Ganança M M, Caovilla HH, M unhoz M SL, Silva M LG, Ganança FF, Ganança CF. Labirintopatias/ labyrinth diseases. Rev Bras Otorrinolaringol 1999; 6(1):3-12.

14. Pedalini MEB, Bittar RSM. Reabilitação vestibular: uma proposta de trabalho. Pró-Fono Rev Atual Cient 1999; 11(1):140-144.

15. Silveira SR, Taguchi CK, Ganança FF. Análise comparativa de duas linhas de tratamento para pacientes portadores de disfunção vestibular periférica com idade superior a sessenta anos. Acta AWHO 2002; 21(1):100-112.

16. Cowand JL, Wrisley DM, Walker M, Strasnick B, Jacobson JT. Efficacy of vestibular rehabilitation. Otolaringol Head Neck Surg 1998; 118(1):49-54. 
17. M ruzek M, Barin K, Nichols DS, Burnett $C N$, Welling DB. Effects of vestibular rehabilitation and social reinforcement on recovery following ablative vestibular surgery. Laryngoscope 1995; 105(1):86-92.

18. Krebs DE, Gill-Body KM, Riley PO, Parker SW. Double-blind, placebo-controlled trial of rehabilitation for bilateral vestibular hypofunction: preliminary report. Otolaringol Head N eck Surg 1993; 109(4):3541.

19. Topuz O, Topuz B, Ardiç FN, Sarhus M, Ogmen G, Ardiç F. Efficacy of vestibular rehabilitation on chronic unilateral vestibular dysfunction. Clin Rehabil 2004; 18(1):76-83.

20. Corna S, Nardone A, Prestinari A, Galante M, Grasso M , Schieppati M. Comparison of Cawthorne-Cooksey exercises and sinusoidal support surface translations to improve balance in patients with unilateral vestibular deficit. Arch Phys Med Rehabil 2003; 84(8):1173-1184.

21. Badke M B, M iedaner JA, Shea TA, Gove CR, Pyle GM. Effects of vestibular and balance rehabilitation on sensory organization and dizziness handicap. Ann Otol Rhinol Laringol 2005; 114(1 Pt 1):48-54.

22. Albera R, Ciuffolotti R, Di Cicco M, Benedittis $G$, Grazioli I, Melzi G, Pallestrini E, Passali D, Serra A, Vicini C. Double-blind, randomized, multicenter study comparing the effect of betahistine and flunarizine on the dizziness handicap in patients with recurrent vestibular vertigo. Acta Otolaryngol 2003; 123(5):588-593.

23. Paiva AD, Kuhn AM, Baccari M. Sintomas psicológicos concomitantes à queixa de vertigem em 846 prontuários de pacientes otoneurológicos do Ambulatório de Otoneurologia da Universidade Federal de São Paulo - Escola Paulista de M edicina. Rev Bras Otorrinolaringol 2004; 70(4):512-515.

Artigo apresentado em 05/12/2007

A provado em 04/03/2008

Versão final apresentada em 02/06/2008 